\title{
Study of the scalability effect of pipe diameter on horizontal air-water flow
}

\author{
Guilherme G. da Silva*, Felipe J. da Silva, Marcelo S. de Castro
}

\section{Abstract}

This article aims to experimentally study the effect of the pipe diameter in two-phase air-water flows.

\section{Key words:}

air-water flow, two-phase flow, pipe diameter effect, pressure gradient, flow pattern.

\section{Introduction}

This study relates the influence of different pipe diameters, with a fixed length, to the flow characteristics, in a two-phase air-water flow, to not only present new results for large diameter pipes but also confirm previous data. Experiments with three different pipe diameters are being made (1", 2" and 3"). Until now, results show different transition regions for intermittent and stratified patterns, comparing with each pipe diameter and some studies published. Besides, a statistical analysis of pressure drop relates its fluctuation with the flow patterns.

\section{Results and Discussion}

Each of the different tubes used in the experiment have a fixed test section length of $12 \mathrm{~m}$. The pressure drop was measured in a distance of $2.5 \mathrm{~m}$, and there is a visualization section made of an acrylic material installed in the end of the test section. The air was injected at $45^{\circ}$ with a device that injects the air inside the tube. A schematic chart of the experiment bench is below.

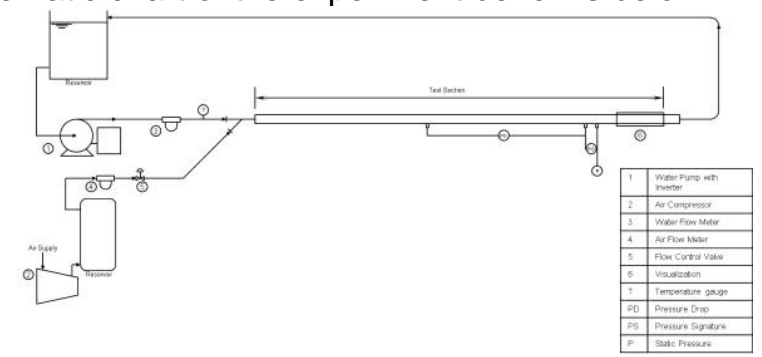

Figure 1. Schematic view of the experiment bench.

About the definition of flow patterns, the different patterns resulting from different mixture velocities were observed. These patterns were registered for each test and were considered the visual patterns definition. Another way used to study the definition of the flow patterns were the measurement of pressure drop in a small section of the pipe, called here as pressure signature [1].

The test procedures were made in two steps: the first one with only water and the second with two-phase flow. The first step is done every day before the two-phase flow tests with a purpose to validate the results of pressure drop in comparison with established theoretical models. After validation, the tests with two-phase flow could get, and it were performed with a fixed water flow rate for which the air flow rate could be varied from the low to upper bench limit. All flow rates were measured three times, in ascending, descending and ascending order, with an acquisition time of 3 minutes, to evaluate hysteresis effects and the benches repeatability, in addition to obtaining statistically relevant data.
As expected, transition boundaries have changed. Comparing with Madhane et al. [2], results for the small diameter shows slugs beginning at lower superficial velocity of water and air, in a transition from "Stratified-Smooth" and "Stratified-Wavy" patterns. Furthermore, the transition from "Elongated-Bubble" to "Slug" begins at lower air superficial velocity. The transition from "Stratified-Smooth" to "Elongated-Bubble" occurs at lower water velocity. Pattern "Stratified-Wavy" also begins for lower air superficial velocity. All the transition regions seem to have shifted left and down. The flow map for $81.3 \mathrm{~mm}$ pipe diameter is plotted in the Fig. 2.

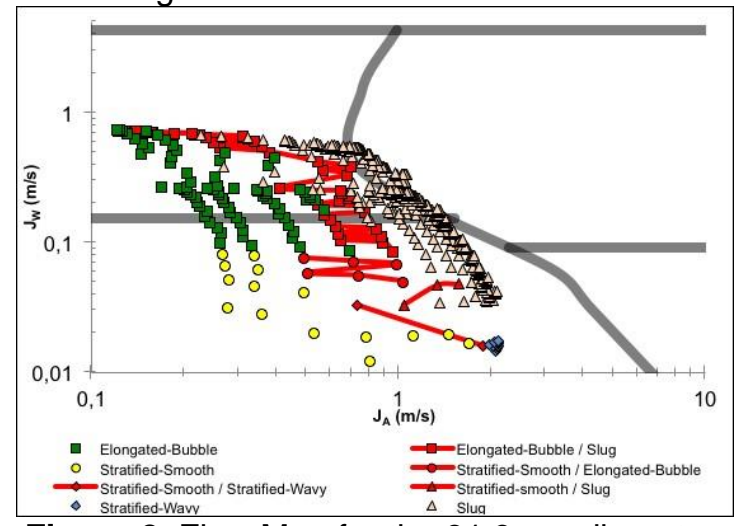

Figure 2. Flow Map for the $81.3 \mathrm{~mm}$ diameter.

\section{Conclusions}

Observed results are approximately in accordance with previous studies that dealt similar theme. Flow pattern maps from Weisman et al. [3] have shown the same transition region displacement observed here, at least for the transition from stratified to intermittent pattern that displaces from lower to greater liquid velocities.

\section{Acknowledgement}

The authors would like to acknowledge CNPq, Alfa Research Group, LABPetro and all of its contributors for the support and suggestions.

[1] Hubbard, M. G., Dukler, A. E.: "The Characterization of Flow Regimes for Horizontal Two-Phase Flow: Statistical Analysis of Wall Pressure Fluctuations", Proceedings of the 1966 Heat Transfer and Fluid Mechanics Institute (1966), 7.

[2] Mandhane, J. M., Gregory, G. A., and Aziz, K.: "Flow-Pattern Map for Gas-Liquid Flow in Horizontal Pipes", Intl. J. Multiphase Flow (1974) 1, 537. [3] Weisman, J., Duncan, D., Gibson, J., and Crawford, T.: "Effect of Fluid Properties and Pipe Diameter on Two Phase Flow Pattern in Horizontal Lines", Intl. J. Multiphase Flow (1979) 5, 437. 